\title{
Monolithic formation of thin films on the vertical surface of a substrate by a dual-ion-beam sputtering technique
}

\author{
Yutaka Uzawa, Sumaru Niida, Masanori Daibo, and Yasuo Kokubun
}

\begin{abstract}
We have developed a novel method for the selective deposition of thin films on the vertical surface of a planar substrate with a vertical step. This was done with a dual-ion-beam sputtering apparatus that is equipped with two ion-beam sources. Using this technique a multilayer filter was monolithically formed on the vertical surface of a Si substrate on which a photodetector had been fabricated, and clear filtering-photodetecting characteristics were observed. This technique can be applied to the monolithic integration of thin-film devices and waveguide-type optical devices with a vertical end facet.

Key words: Integrated photonics, thin film, ion-beam sputtering, multilayer filter, selective deposition.

(c) 1996 Optical Society of America
\end{abstract}

\section{Introduction}

A rf bias-sputtering technique, with which rf power is supplied simultaneously to the target and the substrate, has been proposed to form a smooth film on a rugged substrate. ${ }^{1,2}$ This technique was applied to the deposition of dielectric films on a substrate with a large vertical step of $20-30-\mu \mathrm{m}$ height by a group including one of the authors, and the phenomenon that dielectric films can be selectively deposited on the sidewall of the substrate step, as shown in Fig. 1, was discovered. ${ }^{3-5}$ Since this sidewall is perpendicular to the surface of the substrate, the sidewall of the step is referred to as the vertical surface, otherwise known as the vertical surfaceselective deposition (VSSD) method. ${ }^{5}$ This method is useful for monolithic integration of thin-film devices such as a multilayer filter and waveguide

When this research was done all the authors were with the Department of Electrical and Computer Engineering, Faculty of Engineering, Yokohama National University, 156 Tokiwadai, Hodogayaku, Yokohama 240, Japan. Y. Uzawa is now with the NEC Corporation, 1753 Shimonumabe, Nakaharaku, Kawasaki 211, Japan and M. Daibo is now with Fujikura, Ltd., 1-5-1 Kiba, Koutouku, Tokyo 135, Japan. Y. Kokubun is also with Kanagawa Academy of Science and Technology, 3-2-1 Sakato, Takatsuku, Kawasaki 213, Japan.

Received 5 October 1995; revised manuscript received 22 January 1996.

0003-6935/96/214128-05\$10.00/0

(C) 1996 Optical Society of America devices that have a vertical end facet such as in etched mirror semiconductor lasers. Using this method, a multilayer filter consisting of 17 layers of $\mathrm{TiO}_{2} / \mathrm{SiO}_{2}$ was formed on the vertical surface of a (110) Si substrate, on which a photodetector had been fabricated prior to thin-film deposition, and clear filtering-photodetecting characteristics were observed. ${ }^{4}$

Two problems remain with regard to the shape of the deposited film: one is a steep angle at the top corner of the thin film, and the other is the damage to the top corner of the Si substrate as shown in Fig. 1. In this study we aim to solve these problems by introducing the ion-beam sputtering (IBS) apparatus shown in Fig. 2.

\section{Principle of Vertical Surface-Selective Deposition by the rf Bias-Sputtering Technique}

Let us suppose the deposition and etching of film on a substrate with an inclined slope as shown in the inset of Fig. 3. The target material and $\mathrm{Ar}^{+}$ion are considered to impinge on the substrate almost perpendicularly. The angle of the slope is designated as $\theta$. With rf bias sputtering, the deposition and etching rates have different angular dependences as shown by the dashed curves in Fig. 3. The etching rate has its maximum value around $\theta=40^{\circ}$ because of the so-called oblique incidence effect, and when $\theta=90^{\circ}$ the rate approaches zero. On the other hand, the deposition rate decreases monotonically with an increase of $\theta$, and when $\theta=90^{\circ}$ the rate 


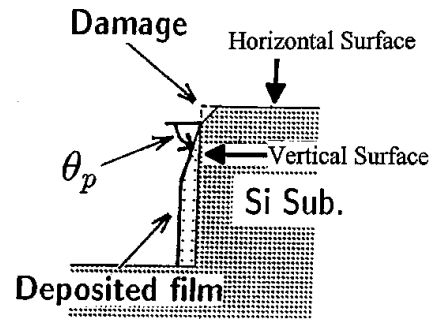

Fig. 1. Problems with the VSSD method when rf bias sputtering was used.

should theoretically be zero. However, it is not actually zero because the target material deposited on the horizontal surface is resputtered by the rf bias power and is redeposited on the vertical surface. ${ }^{3}$

Now let us suppose film deposition on a substrate with a vertical step. In this case there are only two angles on the substrate, i.e., $0^{\circ}$ (horizontal surface) and $90^{\circ}$ (vertical surface). The ratio of $\mathrm{rf}$ powers applied to the target and the substrate can be controlled so that the deposition rate is balanced with the etching rate on the horizontal surface $\left(\theta=0^{\circ}\right)$ and is greater than the etching rate on the vertical surface $\left(\theta=90^{\circ}\right)$ as shown by the dashed curves in Fig. 3. Then a film can be selectively formed on the vertical surface.

However, there have been two problems as shown in Fig. 1. The first is a steep angle (referred to as the peculiar angle $\theta_{p}$ ) that is caused by the existence of an angle at which the deposition rate is balanced with the etching rate as well as $\theta=0^{\circ}$ as shown in Fig. 3. Once the surface with this angle appears, the cross-sectional shape of the film deposited on the vertical surface is limited to the shape of a trapezoid bounded by horizontal and vertical lines and peculiar angle $\theta_{p}$. Therefore, the existence of the peculiar angle reduces the available flat area of the film. The second problem, damage to the top corner of the substrate, is caused by the concentration of the electric field on the top corner. Since the surface of the substrate has a uniform electric potential, the electric field is more likely concentrated on a sharp edge such as the top corner of the step. Because the plasma exists near the substrate with rf bias sputtering, the $\mathrm{Ar}^{+}$ion impinges on the top corner more frequently than on the other part along this concentrated electric field.

To solve these problems, we introduced a dual-ionbeam sputtering apparatus.

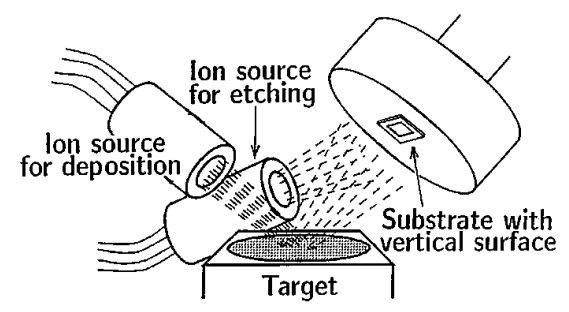

Fig. 2. Schematic configuration of the IBS apparatus.

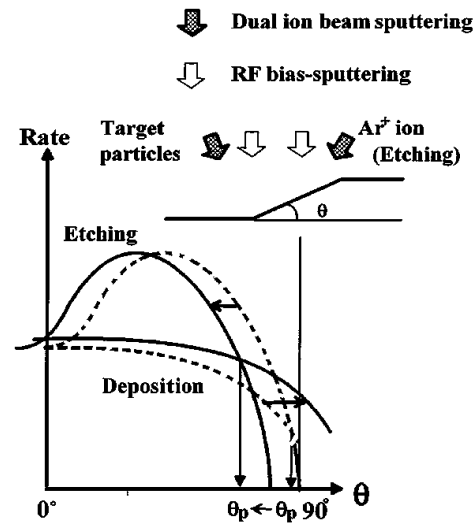

Fig. 3. General deposition and etching characteristics versus slope angle of the substrate. The dashed curves correspond to the $\mathrm{Ar}^{+}$ion and target particles that impinge on the substrate perpendicularly, as indicated by the open arrows in the inset. The solid curves correspond to the $\mathrm{Ar}^{+}$ion that is incident in the direction from upper right to lower left and target particles that are incident in the direction from upper left to lower right, as indicated by the meshed arrows in the inset.

\section{Principle of Vertical Surface-Selective Deposition by the lon-Beam Sputtering Technique}

The IBS apparatus used in this study has two ion sources (a Kaufman-type, 30-mm $\phi$ ion gun made by Commonwealth Scientific Corp.) as shown in Fig. 2. One of the ion sources is irradiated to the target and the sputtered particles are deposited on the substrate. The other is irradiated directly to the substrate. To prevent the accumulation of positive electric charge on the target and substrate, we neutralized these ion beams by using thermal electron radiation just after the beams are emitted from the sources.

With the IBS apparatus, the direction of each ion beam can be varied independently and arbitrarily. This means that the characteristic curves of deposition and etching rates shown in Fig. 3 can be shifted in the lateral direction by changing the incident directions of the beams of target particles and $\mathrm{Ar}^{+}$ ions. When we shift the $\mathrm{Ar}^{+}$ion to the clockwise direction and the target particles to the counterclockwise direction as shown by the meshed arrows in the inset of Fig. 3, the characteristic etching rate curve shifts to the right and that of the deposition rate shifts to the left as shown by the solid curves in Fig. 3 . Therefore, by changing the irradiation angle, we can selectively deposit a thin film on a vertical surface and can reduce the peculiar angle. In addition, the top corner of the substrate is protected from damage because the electric potential of the substrate is kept at zero and there is no plasma near the substrate.

\section{Fabrication of Multilayer Filters}

The vertical surface was formed by an anisotropic wet etching of a (110) Si substrate using an APW etchant (ethylenediamine:pyrocatechol: water $=10$ / 
Table 1. Operating Conditions of the IBS Apparatus

\begin{tabular}{lll}
\hline \multicolumn{1}{c}{ Conditions } & \multicolumn{1}{c}{$\begin{array}{c}\text { Ion Source } \\
\text { for Etching }\end{array}$} & \multicolumn{1}{c}{$\begin{array}{c}\text { Ion Source } \\
\text { for Deposition }\end{array}$} \\
\hline $\begin{array}{l}\text { Beam current } \\
\text { Beam voltage }\end{array}$ & $5 \mathrm{~mA}$ & $20 \mathrm{~mA}$ \\
Background pres- & $1.33-2.67 \times 10^{-4} \mathrm{~Pa}$ & $\begin{array}{l}750 \mathrm{~V} \\
\text { sure }\end{array}$ \\
$\begin{array}{l}\text { Operating pressure } \\
\text { Incident angle on } \\
\text { substrate }\end{array}$ & $4.00 \times 10^{-2} \mathrm{~Pa}$ & $4.00 \times 10^{-2} \mathrm{~Pa}$ \\
$\begin{array}{l}\text { Distance between } \\
\text { ion source and } \\
\text { center of substrate }\end{array}$ & $120 \mathrm{~mm}$ & $90 \mathrm{deg}$ \\
$\begin{array}{l}\text { Distance between } \\
\text { ion source and } \\
\text { center of target }\end{array}$ & & \\
$\begin{array}{l}\text { Distance between } \\
\text { center of target } \\
\text { and center of sub- } \\
\text { strate }\end{array}$ & & $90 \mathrm{~mm}$ \\
\hline
\end{tabular}

17:2/17:5/17 mole fraction, $100{ }^{\circ} \mathrm{C}$ ). The height of the vertical step was approximately $30 \mu \mathrm{m}$.

The operating setup of the IBS apparatus that was used in this experiment is shown in Table 1. Prior to selective deposition on a vertical surface, we deposited $\mathrm{SiO}_{2}$ film on a Si substrate and observed the distribution of film thickness on the horizontal surface. Since the deposition and etching profiles on the horizontal surface are not similar to each other, a balanced region appears i.e., the etching rate is balanced with the deposition rate. This region appears as a band. The substrate with a vertical surface was located in the balanced region. The vertical surface faced the ion beam for etching.
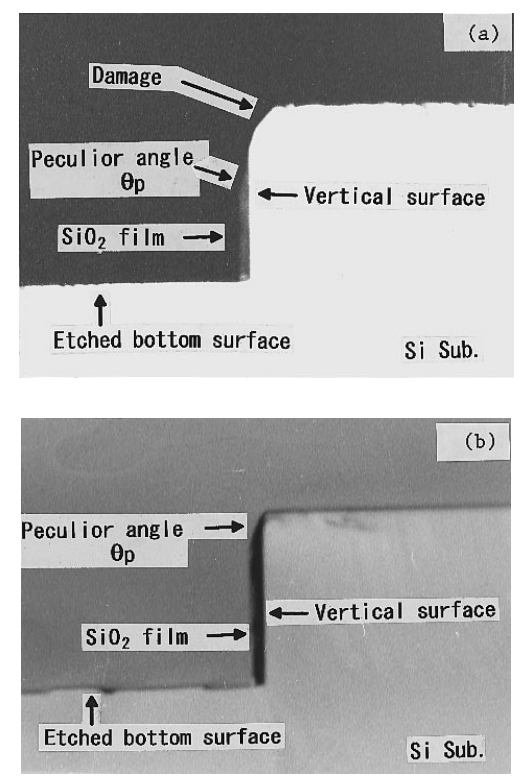

Fig. 4. Optical microscope photographs of a cross section of $\mathrm{SiO}_{2}$ films deposited by (a) rf bias sputtering (Ref. 3) and (b) dual-ionbeam sputtering (this paper).

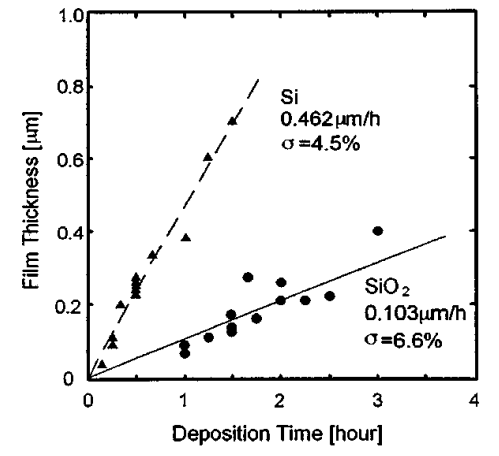

Fig. 5. Measured results of deposition rates of $\mathrm{SiO}_{2}$ and $\mathrm{Si}$ on a vertical surface for which the conditions are listed in Table 1.

With regard to the balanced region, $\mathrm{SiO}_{2}$ film was formed selectively on the vertical surface. An optical microscope photograph of a cross section of the deposited film is shown in Fig. 4(b). Compared with the previous result shown in Fig. $4(\mathrm{a}),{ }^{3}$ which was deposited by the rf bias-sputtering technique, it can be seen that the top corner of the step of the substrate was perfectly protected from damage and the peculiar angle $\theta_{p}$ at the top corner of the film decreased from $75^{\circ}$ to $55^{\circ}$. The deposition rates for $\mathrm{SiO}_{2}$ and $\mathrm{Si}$ on the vertical surface were measured by changing the deposition time. The deposition rates were evaluated to be $0.10 \mu \mathrm{m} / \mathrm{h}$ for $\mathrm{SiO}_{2}$ and 0.46 $\mu \mathrm{m} / \mathrm{h}$ for $\mathrm{Si}$, as shown in Fig. 5 .

To investigate the quality of the film deposited by this method, we formed a multilayer filter on the vertical surface of substrate and measured the filtering characteristic. A simple band-cut filter consisting of four layers of $\mathrm{SiO}_{2}-\mathrm{Si}$ was adopted for this preliminary demonstration. The film thicknesses were designed to be $0.925 \mu \mathrm{m}$ for $\mathrm{SiO}_{2}$ and $0.395 \mu \mathrm{m}$ for $\mathrm{Si}$, which correspond to seven quarters of the cavity wavelength at a source wavelength of 0.83 $\mu \mathrm{m}$. Prior to the formation of a multilayer filter, a pn junction as a photodetector was formed on the vertical surface by diffusing phosphorus into a $p$-type Si substrate. A cross-sectional scanning electron microscope view of the filter is shown in Fig. 6, from which we can observe that the thicknesses of these

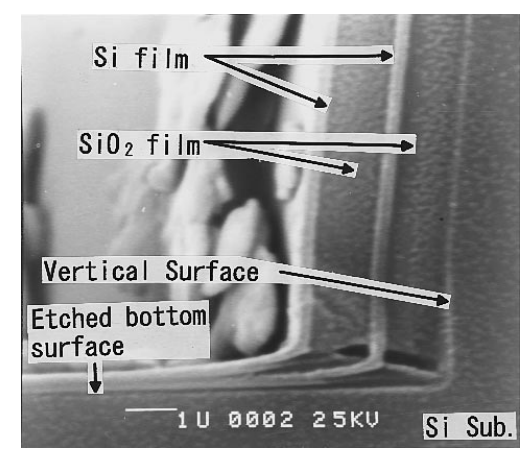

Fig. 6. Scanning electron microscope photograph of four $\mathrm{SiO}_{2}-\mathrm{Si}$ films formed on a vertical surface with a photodetector. 


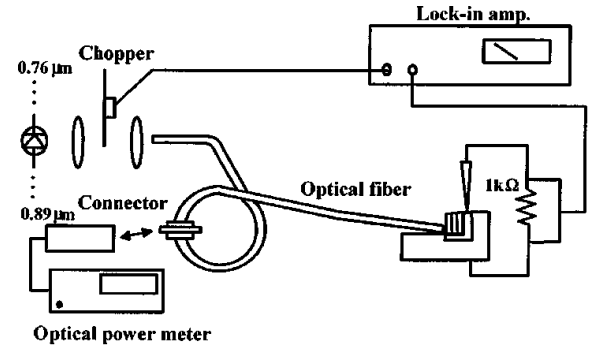

Fig. 7. Experimental setup that was used to measure the filtering-photodetecting characteristics.

films were measured to be $1.1 \mu \mathrm{m}$ for $\mathrm{SiO}_{2}$ and 0.27 $\mu \mathrm{m}$ for Si.

\section{Measurement of Multilayer Filters}

The measurement setup of the filtering-photodetecting characteristics is shown in Fig. 7. Nine laser diodes, lasing at wavelengths ranging from 0.76 to $0.89 \mu \mathrm{m}$, were used as light sources. The laser light was coupled to a single-mode fiber with a connector and the coupled power was monitored at the connector located at the middle point of the fiber. The output end of this fiber was etched to $20-\mu \mathrm{m}$ diameter and was butt-coupled to the multilayer filter.

Clear filtering-photodetecting characteristics were observed as shown by the filled circles in Fig. 8. These measured values are normalized by the wavelength dependence of detector sensitivity that was measured independently. We calculated the theoretical filtering characteristic from actual thicknesses that were measured as shown in Fig. 6 . The refractive-index value of Si film $\left(n_{\mathrm{Si}}=3.67\right)$ and the measured refractive index of $\mathrm{SiO}_{2}$ film $\left(n_{\mathrm{SiO}_{2}}=1.57\right.$ at $0.633-\mu \mathrm{m}$ wavelength) as deposited on the horizontal surface were obtained from Ref. 6. The calcu-

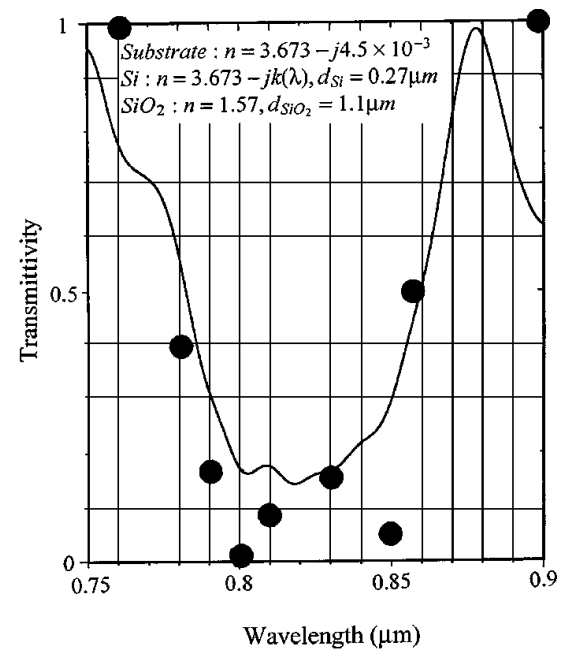

Fig. 8. Theoretical (solid curve) and measured (filled circles) filtering-photodetecting characteristics of a multilayer filter/photodetector integrated device. The absorption coefficient of the Si layer $k(\lambda)$ was taken into account using the published data. ${ }^{6}$ lated values, shown by the solid curve in Fig. 8, agreed well with the measured values. Therefore, we can confirm that the refractive indices of deposited films are almost the same as those of films deposited on the horizontal surface, which were used in our design and analysis. This conclusion is supported by the fact that the wet etching rates of films deposited on a vertical surface are almost the same as those of films deposited on a horizontal surface.

The refractive index of $\mathrm{SiO}_{2}$ film deposited on the vertical surface was measured directly using another sample. To increase the height of the vertical surface, we glued a 2-mm-wide, 10-mm-long Si bar perpendicular to another Si substrate and deposited a $\mathrm{SiO}_{2}$ film selectively on the vertical surface of the $\mathrm{Si}$ bar using the VSSD method. After deposition, the Si bar was removed from the substrate and the refractive index of the $\mathrm{SiO}_{2}$ film was measured using an ellipsometer at 0.633 - $\mu \mathrm{m}$ wavelength for which the result was 1.548 . This value is $1.4 \%$ lower than the 1.57 refractive index of $\mathrm{SiO}_{2}$ film deposited on the horizontal surface and is $6 \%$ higher than that of bulk $\mathrm{SiO}_{2}$. The discrepancy in these values from that of bulk $\mathrm{SiO}_{2}$ was caused by a lack of oxygen, because we did not introduce $\mathrm{O}_{2}$ gas during deposition.

\section{Summary}

We have developed a novel method for the selective deposition of thin films on the vertical surface of a substrate by using a dual-ion-beam sputtering technique. Compared with a rf bias-sputtering technique, we can confirm that the top corner step of the substrate was not damaged and the flat area of the deposited film was increased. In addition, we have demonstrated an integrated device that consists of multilayer filters and a photodetector using this technique and have confirmed that the refractive indices of films on a vertical surface were almost the same as those of films on a horizontal surface. This technique can be applied to the monolithic integration of multilayer filters on the vertical surface of a waveguide end facet.

This research was partly supported by grant-inaid 02452174 from the Ministry of Education and Culture and the Mitsubishi Foundation. The authors express their sincere thanks to Sai Tak Chu for help with the calculation of the theoretical curve in Fig. 8.

\section{References}

1. C. Y. Ting, V. J. Vivalda, and H. G. Schaefer, "Study of planarized sputter-deposited $\mathrm{SiO}_{2}$," J. Vac. Sci. Technol. 15, 1105-1112 (1978).

2. T. Mogami, M. Morimoto, H. Okabayashi, and E. Nagasawa, " $\mathrm{SiO}_{2}$ planarization by two-step rf bias-sputtering," J. Vac. Sci. Technol. B 3, 857-861 (1985).

3. S. Tamura, T. Baba, and Y. Kokubun, "Selective formation of dielectric films on vertical surface of substrate for photonic integrated circuits," IEEE J. Quantum Electron. 28, 17271731 (1992).

4. T. Baba, S. Tamura, Y. Kokubun, and S. Watanabe, "Monolithic 
integration of multilayer filter on vertical surface of semiconductor substrate by bias-sputtering technique," IEEE Photon. Technol. Lett. 2, 191-193 (1990).

5. S. Watanabe, M. Takizawa, and Y. Kokubun, "Demultiplexer/detector monolithic integrated device by vertical surface selective deposition (VSSD) method," in Proceedings of 17th European Conference on Optical Communication/8th International Conference on Integrated Optics and Optical Fiber Communication (Société des Electriciens et des Electroniciens, Paris, 1991), paper TuPS1.27.

6. Properties of Silicon, EMIS Dataview Series No. 4, (Institution of Electrical Engineers, London, 1988), pp. 72-78. 